\title{
Freiheit durch Partizipation. Ein Oxymoron?
}

\author{
Christian Swertz, Universität Wien
}

April 2013

\begin{abstract}
Der Partizipationsbegriff scheint durch seine ubiquitäre Verwendung an medien- und gesellschaftskritischem Gehalt und damit an Relevanz für die Medienpädagogik zu verlieren. Im Beitrag wird diesem Bedeutungsverlust nachgegangen und vor diesem Hintergrund einen Vorschlag für eine kritische Profilierung des Partizipationsbegriffs entwickelt.
\end{abstract}

Keywords: Partizipation, Medienkompetenz, Freiheit

\section{Einleitung}

Der Ausdruck Partizipation wird in der pädagogischen Fachdiskussion häufig verwendet. Auch wenn die Aussagekraft einer einfachen Stichwortsuche ohne bibliometrische Analyse gering ist, kann der Umstand, dass eine Stichwortsuche nach „participation“ in der Literaturdatenbank des „Education Ressources Information Center“ 76.772, eine Stichwortsuche nach „Partizipation“ im „Fachinformationssystem Bildung“ 3.555 und im „Informationssystem Medienpädagogik“ 989 Nachweise liefert, als erster Hinweis auf den breiten Gebrauch des Ausdrucks „Partizipation“ gesehen werden.

Dabei legt eine erste, notwendig unvollständige Durchsicht den Eindruck nahe, dass der Ausdruck insgesamt meist weniger als spezifischer Begriff, sondern eher als unspezifische, aber stets positiv konnotierte Bezeichnung verwendet wird. Etwas überspitzt formuliert könnte eine Sichtung der ver- 
fügbaren Quellen zusammengefasst werden mit: Alle sollen sich irgendwie an allem beteiligen: Schüler sollen sich an der Schule beteiligen, Eltern ebenso, ehemalige Soldatinnen und Soldaten an Beratungskursen, Lernende am Sportunterricht, Studierende an kooperativen Methoden in universitären Seminaren, Interviewpartnerinnen und Interviewpartner an Gruppentreffen von Forscherinnen und Forschern, Lernende an Bibliotheken, Einwanderer an universitären Studienprogrammen, Menschen mit verschiedenen Formen von Beeinträchtigungen an ebenso verschiedenen Formen von Bildungsprogrammen, Bürgerinnen und Bürger an staatlicher Administration etc. Wer sich warum wie woran beteiligen soll, spielt dabei kaum eine Rolle. Auch klassische Differenzen lassen sich kaum feststellen: Als Partizipation werden gleichermaßen die staatlich geforderte Beteiligung der Bürgerinnen und Bürger an eGovernance, die Förderung der Beteiligung Jugendlicher aus sozial benachteiligten Schichten an der Gesellschaft und die Beteiligung politischer Bewegungen an demokratischen Entscheidungen bezeichnet.

Die breite Verwendung des Ausdrucks „Partizipation“ ist ein interpretationsbedürftiges Phänomen. Für diese Interpretation ist es hier naheliegend, zunächst eine medientheoretische Perspektive einzunehmen. Aus einer an die Toronto School angelehnten Sichtweise ist der Umstand, dass vielen Menschen Beteiligung als relevant erscheint, nicht überraschend, sondern spiegelt die in Relation zu Buchdruck und Fernsehen kalten Eigenschaften der Computertechnologie wieder. Der Einfluss eines relativ kalten Mediums fördert, wie McLuhan argumentiert hat, eine relativ heiße, d.h. involvierende, beteiligte und d.h. hier partizipative Kultur (Swertz 2000). Da Computertechnologie inzwischen in allen Bereichen der hier zu berücksichtigenden Gesellschaften zum dominanten Medium geworden ist, also als zentrale Kulturtechnik zu verstehen ist (Meder 1998), ist es nicht überraschend, dass Partizipation oft als etwas Positives akzeptiert wird. Die breite Akzeptanz des Ausdrucks Partizipation entspricht einer dominanten Nutzung der Computertechnologie als Medium menschlicher Verständigung.

Die häufige Verwendung in wissenschaftlichen Arbeiten erinnert daran, 
dass die involvierende Tendenz der relativ kalten Computertechnologie auch die im Wissenschaftssystem arbeitenden Wissenschaftlerinnen und Wissenschaftler, die diese Tendenz nicht nur in der breiten Verwendung des Ausdrucks Partizipation, sondern auch in der Akzeptanz für die Verwendung partizipativer Forschungsmethoden zum Ausdruck bringen, betrifft. Dieser Umstand kann nun mangels einer jenseits solcher Tendenzen liegenden Position nicht als gut oder böse schlechthin ausgewiesen werden, sondern allenfalls aus Sicht einer an ein anderes Medium gebundenen Position reflektiert werden.

Dieses wissenschaftstheoretische Problem wird im Folgenden nicht in den Mittelpunkt gerückt, weil der Umstand, dass die wissenschaftliche Verwendung von Ausdrücken innerhalb einer Gesellschaft stattfindet, die wiederum ihren Eindruck in diesen Ausdrücken hinterlässt, weder als neu noch als überraschend angesehen werden kann. Relevanter ist hier der Umstand, dass ein Medium von Menschen mittels des Mediums auch bezeichnet werden kann. Eine Änderung des gesellschaftlich dominanten Mediums kann so zwar nicht legitimiert werden, möglich wäre es aber, sich für die Teilhabe an einer anderen, nicht von Computertechnologie durchdrungenen (Sub-)Kultur zu entscheiden oder eine Präferenz für spezifische Verwendungsformen zu praktizieren.

Diese Möglichkeit wird für die Medienpädagogik derzeit nicht zuletzt deswegen relevant, weil mit der Verbreitung der Computertechnologie zugleich die Tendenz zu einem reflexiven Denken verbunden ist (Meder, Swertz), die mit den medialen Brüchen zwischen den vielen mit universellen Turingautomaten sprachlich erzeugten Medien einhergeht. Das ermöglicht es nicht, eine letztbegründete Position zu diesen Tendenzen einzunehmen (diese Idee entspricht eher dem Buchdruck); es ist aber nicht ausgeschlossen, eine mit dem Gehirn als Medium des Denkens verbundene Willensentscheidungen ermöglichende Position gegenüber medialen Tendenzen einzunehmen. Das erlaubt zumindest die mit dieser Bemerkung zugleich in einem ersten Punkt erreichte Exposition der jeweiligen Position. 
Die Eingangs skizzierte breite Verwendung des Ausdrucks „Partizipation“ sowie die damit verbundenen medialen und gesellschaftlichen Tendenzen legen es zunächst nahe, den Ausdruck „Partizipation“ als Bezeichnung für das Gute und Wahre in der Wissensgesellschaft zu verstehen. Aus dieser Sicht ist es zunächst zu empfehlen, den Ausdruck Partizipation häufig zu verwenden, weil Leserinnen und Leser, Gutachterinnen und Gutachter oder Politikerinnen und Politiker alles, was als Partizipation bezeichnet wird, akzeptieren werden. Überspitzt gesagt: Partizipation erfordert derzeit die Verwendung des Wortes „Partizipation“, das in dieser Hinsicht als Modebegriff charakterisiert werden kann. Unklar ist, ob mit einem solchen Modebegriff jenseits der zumindest als Reflexionsanlass dankbaren breiten Verwendung ein Begriff bezeichnet werden kann, der für die medienpädagogische Theoriebildung relevant ist.

Für die bereits skizzierten medialen Tendenzen ist das jedenfalls nicht der Fall, weil diese in medienkritischer Absicht bereits als involvierende Tendenz kalter Medien bezeichnet werden können. Die ubiquitäre Verwendung des Partizipationsbegriffs legt aber auch die Vermutung nahe, dass sie Ausdruck einer Aneignung des positiv besetzten Begriffs durch Herrschende ist. Die ubiquitäre Verwendung wäre dann als ein Ausdruck des Eindrucks von Machtinteressen zu verstehen. Das würde den möglichen medienpädagogische Sinn des Partizipationsbegriffs zugunsten einer standardisierenden Verwendung suspendieren. Daher soll hier der Versuch unternommen werden, ausgehend von vorliegenden Arbeiten einen medien- und gesellschaftskritischen Partizipationsbegriff zu identifizieren, der in der Medienpädagogik sinnvoll verwendet werden kann.

Diese Identifikation soll hier nicht durch eine axiomatische oder prinzipientheoretische Entfaltung des Begriffs gewonnen werden, sondern durch ein sowohl fragmentarisches als auch Akzeptanz erforderndes Spiel mit Perspektiven. Dazu werden hier in nicht ganz, aber auch beliebiger Auswahl die unterrichtsmethodische, die ökonomische und die politische Perspektive eingenommen. Dabei steht die Frage, wer wie woran partizipierensoll, in- 
wiefern also mit dem Partizipationsbegriff eine normative Orientierung für die mit dem Medienkompetenzbegriff diskutierte Fachdidaktik Medien erreicht werden kann, im Mittelpunkt.

\section{Perspektiven}

\subsection{Methodik}

Ein erster Begriff, der als Partizipation bezeichnet wird, ist im Bereich der Unterrichtsmethoden angesiedelt. Exemplarisch für diese Perspektive werden hier die Arbeiten von Schaumburg (2011), Grell/Rau (2011) und Wijnen (2012) herangezogen.

Schaumburg diskutiert die Potenziale von Web-2.0-Medien für die politische Bildung und evaluiert die Nutzung dieser Potentiale aus Sicht der Nutzerinnen und Nutzer. Dabei wird zunächst betont, dass die Web-2.0-Medien „,im Vergleich zum Web 1.0 ein erheblich größeres Potenzial zur Mitgestaltung politischer Inhalte und Prozesse und bzw. zur Partizipation bieten“ (Schaumburg 2011: 1). Schaumburg untersucht allerdings nicht die politische Praxis, sondern die politische Bildung. In der politischen Bildung kann aber, wenn diese von der politischen Praxis unterschieden wird, nur eine Auseinandersetzung mit den im Rahmen der politischen Bildung in einen Unterrichtsgegenstand transformierten (Gruschka 2002) Gestaltungen politischer Inhalte und Prozesse stattfinden. Partizipation bezeichnet dann nicht die Beteiligung an politischen Prozessen, sondern die Beteiligung am Unterricht.

Schaumburg betont weiter als herausragende Eigenschaft der Web-2.0Medien, „dass sie prinzipiell jedem Internetnutzer die Möglichkeit eröffnen, Informationen öffentlich zu erstellen, zu verändern oder zumindest zu kommentieren“ (Schaumburg 2011: 2). Das Gleiche kann auch von visuellen Medien wie der Zeitung oder auditiven Medien wie dem Bürgerfunk behauptet werden. Ein grundsätzlicher „Wandel in den Interaktions- und Parti- 
zipationsmöglichkeiten“ (Schaumburg 2011: 2) ist also nicht von vornherein anzunehmen, sondern wäre erst zu zeigen.

Empirisch untersucht wird von Schaumburg dann nicht die Beteiligung von Akteurinnen und Akteuren an politischen Prozessen, sondern die Zufriedenheit von Teilnehmerinnen und Teilnehmern an Bildungsangeboten. Dabei wird gezeigt, dass die Inhalte und die Arbeit der Lehrenden von den Teilnehmerinnen und Teilnehmern als relevanter angesehen werden als die verwendeten Web 2.0-Medien. Die Notwendigkeit, Partizipation als neuen Begriff einzuführen, wird dabei nicht deutlich.

Exemplarisch sichtbar wird bei Schaumburg, dass Medienpädagoginnen und Medienpädagogen ihre Aufmerksamkeit derzeit häufig auf die Computertechnologie und dabei insbesondere auf die Software und die Praktiken, die unter der Bezeichnung Web 2.0 zusammen gefasst werden (vgl. zu diesem Begriff Panke 2007), richten. Es entsteht der Eindruck, dass hier vor allem der Umstand des Neuen fasziniert (Swertz 2006). Das kann als Spiegelung der durch Computertechnologie forcierten dynamischen Strukturen der Gesellschaften, in denen die Autorinnen und Autoren leben, verstanden werden. In diesem Sinne wäre eine Handlung schon dann als Ausdruck einer sinnvollen und wünschenswerten Partizipation zu verstehen, wenn sie mit Hilfe einer noch nicht allzu lange verfügbaren Software, die irgend eine Form menschlicher Kommunikation in Computernetzwerken ermöglicht, erfolgt. Während das Eigenrecht einer solchen Ausbildung in seiner Relevanz nicht zu unterschätzen ist, würde die Beschränkung darauf ebenso relevante Aspekte pädagogischen Bemühens von vornherein suspendieren.

Ein anderes Handlungsfeld wird in der Arbeit von Grell und Rau betrachtet. Sie diskutieren in ihrem Übersichtsartikel Arbeiten zur Partizipation Studierender an der Hochschullehre mit Web-2.0-Medien. Im Mittelpunkt steht dabei das „Phänomen ausbleibender studentischer Partizipation“ (Grell/Rau 2011: 1). Für dieses Phänomen liefern die von Grell und Rau referierten Beiträge verschiedene Erklärungen: Der geringe Neuigkeitscharakter der Web-2.0-Medien, die Entstehung von Massenuniversitäten, die pragmati- 
sche Haltung der Studierenden, die mangelnde didaktische Planung und die Institutionalisierung von Bildungsprozessen.

Nicht geklärt wird jedoch, welcher Begriff dabei mit Partizipation bezeichnet wird. Es finden sich lediglich verschiedene Formulierungen wie Partizipationsmöglichkeiten, aktivere Partizipation, Partizipationsaspekte von Akteuren in formalen Bildungssettings, Partizipationsgrade oder die unfreiwillige Partizipation. Lediglich an einer Stelle findet sich die Beschreibung einer aktiven Mitgestaltung von Erkenntnisprozessen, die als ,relevant für die Entfaltung der eigenen Bildungsprozesse und Kompetenzentwicklung " $\mathrm{zu}$ sehen ist (Grell/Rau 2011: 17). Wie es angesichts zunächst ausbleibender und dann unfreiwilliger Partizipation allerdings zu einer Freiheit bei all dem Zwange kommen könnte, wird in den von Grell und Rau referierten Arbeiten offenbar nicht diskutiert.

An anderen Stellen werden Handlungen wie Äußerungen in Blogs oder Wikis als Beispiele für partizipatorische Handlungen genannt. Solche Handlungen wurden bisher als Mitarbeit im Unterricht bezeichnet. Implizit ist damit in der Regel die mündliche Mitarbeit gemeint. Diskutiert wird dabei derzeit vor allem das Problem der Beurteilung der mündlichen Mitarbeit (etwa bei Nickel 2004 oder Kirk 2009). Dieses Problem stellt sich offenbar auch im Zusammenhang mit Web-2.0-Medien.

Mündliche Mitarbeit kommt bisher häufig im Unterrichtsgespräch vor. Das nach wie vor in vielen Unterrichtsformen und -fächern relevante Unterrichtsgespräch (vgl. dazu z.B. Mückel 2011 oder Mehr 2010) ist keine neue Erfindung, sondern wird einschließlich heute noch gebräuchlicher Praktiken wie z.B. der an die Lernenden gerichtete Frage: „Was habe ich eben gesagt?" bereits von Comenius in der Didacta Magna als wichtiges didaktisches Mittel im Frontalunterricht vorgeschlagen. Diese Form, die Mitarbeit im Unterrichtsgespräch des Frontalunterrichts, scheint bei den von Grell/Rau referierten Praktiken im Mittelpunkt zu stehen. Andere Praktiken, wie die ebenfalls gut etablierten Formen der Projekt- oder Gruppenarbeit, werden jedenfalls nicht angedeutet. 
Nun wurde das Medium geändert, denn in den von Grell und Rau referierten Ansätzen steht nicht die mündliche Mitarbeit, sondern die schriftliche Mitarbeit in Web-2.0-Medien im Mittelpunkt. Das kann auch als Online-Mitarbeit bezeichnet werden, die bei Verwendung entsprechender Medien durchaus auch mündliche Mitarbeit sein könnte. Dieser Medienwechsel mag zu Veränderungen führen, die es sinnvoll erscheinen lassen, den zur Rede stehenden Sachverhalt nicht als Online-Mitarbeit, sondern als Partizipation zu bezeichnen.

Allerdings weisen die von Grell/Rau berichteten Probleme darauf hin, dass die Ähnlichkeiten die Unterschiede überwiegen. Schon die Zwangsstruktur ist im Präsenzunterricht ähnlich. So gibt es durchaus auch in Präsenzseminaren die Situation, dass nur wenige Studierende sich beteiligen. Zwangsmaßnahmen werden für Präsenzseminare zwar seltener empirisch untersucht, aber schon eine Teilnahmepflicht oder die Praxis, Teilnehmende, die von sich aus überhaupt kein Interessen an einer mündlichen Mitarbeit zeigen, durch direkte Ansprache zur Mitarbeit zu nötigen, weisen auf Parallelen hin. Ein weiterer Aspekt ist die von Grell/Rau betonte Relevanz der Partizipation als aktive Mitgestaltung von Erkenntnisprozessen für die Entfaltung der eigenen Bildungsprozesse und Kompetenzentwicklung. Geht man etwa dem Ausdruck ,,aktive Mitgestaltung“ nach, so ist zunächst klar, dass Lern- oder Bildungsprozesse nicht einfach nur passiv sein können, sondern immer eine Antwort des Lernenden impliziert (Mitgutsch 2008). Auch für die aktive Mitgestaltung finden sich in der Didaktik zahlreiche Beispiele, so dass hier der Hinweis auf den Ansatz von Dewey (2000) und Klafki (1985) genügen kann.

Es erscheint also weder als sinnvoll, das bisher als Mitarbeit bezeichnete didaktische Prinzip nun als Partizipation zu bezeichnen, noch die mediendidaktische Aufgabe, Formen der Mitarbeit für verschiedene Medien zu konzipieren und zu untersuchen, so zu benennen. Vielmehr erscheint es angesichts von Praktiken erzwungener Mitarbeit, die als Nötigung zur Partizipation zu verstehen wären, bestenfalls als Karikatur einer möglichen medien- 
pädagogischen Absicht, hier den Ausdruck Partizipation zu verwenden.

Eine dritte Perspektive wird von Wijnen in den Mittelpunkt gerückt. Sie diskutiert die in der Fachdidaktik Medien prominente Methode der handlungsorientierten Medienpädagogik am Beispiel von Community Medien: „Community Medien drehen den Spieß um - aus Rezipientinnen und Rezipienten von Fernseh- und Radiosendungen werden aktive Sendungsmacherinnen und Sendungsmacher, die eigene Inhalte produzieren und ihre Gedanken, Anliegen und Ideen der Öffentlichkeit zugänglich machen. Sie ermöglichen aktive und kreative Medienarbeit und sind somit auch aus medienpädagogischer Perspektive von besonderer Bedeutung, da sie auf unterschiedlichen Ebenen zur Förderung von Medienkompetenz beitragen können“ (Wijnen 2012).

Dabei geht es Wijnen nicht um die Transformation einer politischen Medienbewegung in eine außerschulische Bildungsinstitution, die vollzogen wäre, wenn die Förderung von Medienkompetenz zum Hauptanliegen des Bürgerfunks werden würde, weil dann die Mitarbeit im Bürgerfunk nicht als politische Handlung, sondern als pädagogische Maßnahme zu verstehen wäre. Klar ist aber, dass die von Wijnen treffend beschriebene aktive und kreative Medienarbeit ohne weiteres als informelle Bildung gefasst werden kann, was auch in entsprechenden Zertifizierungen zum Ausdruck kommt (z.B. VHS Münster 2012). Das Verständnis als informelle Bildung wird auch daran deutlich, dass als Ziel die Förderung von Medienkompetenz und nicht die politische Handlungsfähigkeit gesehen wird. Während dieser Ansatz eine hohe Relevanz für die Fachdidaktik Medien hat, gibt es wenig Anlass, dies als Partizipation zu bezeichnen.

\section{2 Ökonomie}

Der am 6.9.2012 veröffentlichte Bericht der „Gruppe hochrangiger Sachverständiger der EU für Schriftsprachkompetenz“ ist vermutlich die aktuellste Äußerung der Europäischen Union zu einem medienpädagogischen Thema. In der deutschen Kurzfassung dieses Berichts heißt es einleitend: „Bei der 
Beherrschung der Schriftsprache geht es um das Selbstwertgefühl der Menschen und um die Fähigkeit, als Privatpersonen, aktive Bürger, Arbeitnehmer oder Eltern in der Gesellschaft zu funktionieren und sich darin zu entfalten “ (Gruppe hochrangiger Sachverständiger 2012: 3). Schon in dieser Formulierung irritiert aus medienpädagogischer Sicht der Ausdruck „funktionieren“, der der „Entfaltung“ voran gestellt wird. Zwar könnte mit „funktionieren“ und „entfalten“ an dieser Stelle das traditionell mit dem Verhältnis von Bildung und Ausbildung thematisierte Verhältnis von Fremd- und Selbstbestimmung gemeint sein; mit einem bloßen Funktionieren wäre das aber kaum zu bezeichnen. Genau das steht aber im Mittelpunkt, wie in der Darstellung des identifizierten Problems deutlich wird: „Gute Lese-und Schreibfähigkeiten sind für die Verbesserung der Lebensbedingungen der Menschen und die Förderung eines starken und nachhaltigen Wirtschaftswachstums in Europa von zentraler Bedeutung. “(ebd.: 4). Ähnlich heißt es im Hauptbericht: „Literacy skills are crucial to parenting, finding and keeping a job, participating as a citizen, being an active consumer, managing one's health and taking advantage of digital developments, both socially and at work. " (EU High Level Group of Experts on Literacy: 2012: 21). Die Bedeutung von „manging one's health“ und „taking advantage of digital developments“ ist hier eindeutig: Es geht um den Konsum entsprechender Produkte, nicht aber um die menschliche Freiheit, die in dem 116 Seiten langen Bericht nicht erwähnt wird. Ähnliches gilt für Vorstellungen wie die Selbstbestimmung des Menschen. Unter der Überschrift „Gains for Individuals“ heißt es: „While self-confidence may not have a quantifiable economic value, it stimulates economic and social success through the aspiration to be successful“" (ebd.: 26). Schon der Umstand, dass eine fehlende ökonomische Quantifizierbarkeit bedauert und damit als Maßstab gesetzt wird, macht in Verbindung mit dem Kriterium des Erfolgs deutlich, dass es hier um ökonomischen Erfolg unter konkurrenzkapitalistischen Verhältnissen geht.

Diese Schwerpunktsetzung ist nicht überraschend, da das Konzept ausdrücklich in den Kontext der ausschließlich ökonomisch ausgerichteten 
Agenda „Europa 2020“ (European Commission 2010) gestellt wird. Partizipation kommt in diesem Dokument nur einmal vor: "An agenda for new skills and jobs to modernise labour markets and empower people by developing their of skills throughout the lifecycle with a view to increase labour participation and better match labour supply and demand, including through labour mobility“ (European Commission 2010: 6). Das lässt an Deutlichkeit wenig zu wünschen übrig: Es geht um die Konkurrenzfähigkeit auf dem Arbeitsmarkt. Einen anderen Grund als die Verschleierung der offensichtlichen Absicht, das als Partizipation zu bezeichnen, gibt es nicht. Beschäftigung, Berufstätigkeit oder Arbeitslosigkeit reichen hier aus.

Relevant erscheint allerdings, auch wenn das schon häufiger thematisiert worden ist (vgl. z.B. Dzierzbicka 2006, Lohmann 2010), ein kurzer Exkurs zum neoliberalen Denkansatz, der hinter der Politik der EU-Kommission steht. Mit der nicht zuletzt auf die einflussreichen Arbeiten des Wirtschaftsnobelpreisträgers Hayek (1960) zurückgehende Idee des Neoliberalismus (Gertenbach 2007) wird die Möglichkeit der Konkurrenz in den Mittelpunkt gerückt.

Dabei wird von Hayek ein auf dem Konkurrenzgedanken basierendes ökonomisches Wahrheitssystem als zentrales Wahrheitssystem gesetzt, dem andere Wahrheitssysteme untergeordnet werden. Das ist im hier zur Diskussion stehenden Zusammenhang insbesondere für das politische System sowie das Wissenschaftssystem relevant; mag aber auch für das religiöse und das künstlerische Wahrheitssystem gelten. Eine Legitimation für den Primat dieses einen Wahrheitssystems liefert Hayek jedoch nicht; vielmehr wird seine Position an dieser Stelle widersprüchlich, weil es gerade die sich in Vielfalt ausdrückende menschliche Freiheit ist, die erreicht werden soll, diese aber durch den Primat eines Wahrheitssystems unterlaufen wird. Konsequenter wäre es im ökonomischen Kontext zu fordern, dass es verschiedene (Wirtschafts-)systeme, etwa ein kompetitives und ein kooperatives, nebeneinander geben sollte - aber genau diese Forderung stellt Hayek nicht auf. Ein Ansatz, der ohne die prinzipielle Anerkennung auch anderer möglicher 
Wahrheiten oder zumindest der Möglichkeit des eignen Scheiterns operiert, ist aber dogmatisch.

Analysiert man den über die Forderungen der EU für die Medienpädagogik relevant werdenden neoliberalen Dogmatismus, bietet die Interpretation als Instauration des Manchesterliberalismus ein interessantes Argument: Der Manchesterliberalismus rückt Freihandel in Verbindung mit uneingeschränkter Macht der Kapitalisten über die Arbeitenden in den Mittelpunkt. Sie setzten sich für die Abschaffung von Zöllen und die Einführung des Freihandels ein - und genau das tun die Unternehmen, die das Web 2.0 betreiben, auch, wenn sie etwa die staatliche Kontrolle durch Datenschutzgesetze zu unterlaufen suchen.

Gleichzeitig wurde von den Manchesterkapitalisten vehement gegen Einflüsse des Staates im wirtschaftlichen Bereich gestritten, und zwar insbesondere gegen das Fabrikgesetz, mit dem verboten wurde, dass Kinder und Frauen mehr als 10 Stunden am Tag arbeiten mussten. Dabei wurden die Industriearbeiter für ihre Tätigkeit immerhin entlohnt. Diese Mühe machen sich die Betreibergesellschaften der meisten Web-2.0-Medien nicht. Vielmehr haben Sie einen Weg gefunden, Aufmerksamkeit, die als knappes Gut die zentrale Ware der Informationsgesellschaft ist, ohne Gegenleistung zu lukrieren. Der Verkauf kostenloser Arbeitskraft war aber noch immer ein wirtschaftliches Erfolgsmodell. Das mit Web-2.0-Medien auch noch eine perfide Form von Kinderarbeit realisiert wird, machen aktuelle Nutzungsdaten deutlich. Kurz gesagt, wird damit die Freiheit des Kapitals, nicht aber die Freiheit von Menschen realisiert.

Das hier skizzierte Verständnis von Partizipation wird als Teilhabe an einer Marktkonkurrenz bezeichnet. Dieses Verständnis liefert in einer sich als sozial- und marktwirtschaftlich verstehenden Gesellschaft eine Maßgabe, in der die kompetitive Teilnahme an der Marktkonkurrenz zur Norm wird. Zur Möglichkeit der Teilnahme an der Marktkonkurrenz beizutragen ist dann relevant, wenn die Ausgrenzung von Menschen aus der Marktkonkurrenz als Problem gesehen wird. Nur kann der Umstand der Ausgrenzung aus der 
Marktkonkurrenz mit einer Förderung der Fähigkeit zur Teilhabe an der Marktkonkurrenz nicht nachhaltig bearbeitet werden, weil Ausgrenzung, also Marktaustritte, ein wesentliches Moment des Prinzips konkurrenzkapitalistischen Wirtschaftens ist und daher ein strukturelles Moment darstellen, dass, wenn es denn gelöst werden soll, nur strukturell zu lösen wäre. Aus der hier vertretenen Position heraus kann das zwar bedacht und insofern medienpädagogisch berücksichtigt werden. $\mathrm{Zu}$ einer medienpädagogischen Norm kann es aber nicht gewendet werden. Daher ist die Vermittlung der Kompetenz zum Markteintritt um die Vermittlung der Kompetenz zum Marktaustritt zu ergänzen. Damit rückt auch die Kompetenz zum zufriedenen Leben in einer ausgegrenzten Position, etwa in einer Heterotopie (Foucault 1992) in den Blick.

Aber weder dafür ist der Ausdruck Partizipation erforderlich, noch für den Umstand, dass Facebooknutzerinnen und -nutzer derselben Freiheitsillusion unterliegen wie rechtlose Landarbeiterinnen und Landarbeiter, die glaubten, Freiheit zu gewinnen, indem Sie sich als Industriearbeiterinnen und Industriearbeiter verdingten.

\subsection{Politik}

Der Ausdruck Partizipation wurde von Milbrath (1965) in die Politikwissenschaft eingeführt. Milbrath hat politisch handelnde Menschen als Gladiatorinnen und Gladiatoren beschreiben, über deren Schicksal das Publikum entscheidet, und zum Verständnis der damit verbundenen Praktiken die Beteiligung an Wahlen und Parteien untersucht. Zum Ausgangspunkt für die derzeitige breite Verwendung des Partizipationsbegriffs wird allerdings die „Citizen Participation“, zu deutsch: die Bürgerinnen- und Bürgerbeteiligung, also weniger die unmittelbare, institutionalisierte, konventionelle und legale Partizipation, sondern eher die indirekte, nicht institutionalisierte, unkonventionelle und gelegentlich illegale Partizipation.

Die meisten Arbeiten, die sich mit Partizipation im Sinne der Bürgerinnenund Bürgerbeteiligung beschäftigen, beziehen sich, wie Collins und Ison 
(2006) feststellen, auf die 1969 veröffentlichte „Ladder of Participation“ von Arnstein. Arnstein geht von der Beobachtung aus, dass viele Bürgerbeteiligungsprogramme lediglich die Funktion haben, Beteiligung behaupten zu können, ohne dass aber eine Beteiligung in dem Sinne, dass die Ergebnisse von Entscheidungsprozessen beeinflusst werden, tatsächlich stattfindet. Daher unterscheidet sie verschiedene Formen der Bürgerbeteilung, die nach dem Ausmaß der Effekte der Bürgerbeteiligung auf den Ausgang von Entscheidungsprozessen in Stufen einer Leiter geordnet werden: Manipulation, Therapie, Information, Anhörung, Platzierung, Partnerschaft, Machtdelegation und Bürgerkontrolle. Zur obersten Stufe schreibt Arnstein: At „Citizen Control, have-not citizens obtain the majority of decision-making seats, or full managerial power“" (Arnstein 1969: S. 4).

Nun wurde das Jahr 2013, in dem dieser Artikel formuliert wurde, vom Europäischen Parlament und Rat als „Jahr der Partizipation“ ausgerufen (Europäisches Parlament 2012). Mit Arnstein muss es allerdings durchaus misstrauisch machen, wenn die herrschende politische Elite zur politischen Partizipation einlädt. Denn es wäre überraschend, wenn Menschen, die oft mit hohem ökonomischem, kulturellem und sozialem Kapitaleinsatz Macht errungen haben, diese ohne weiteres und vor allem ohne Machtkampf hergeben. Und in der Tat ist das auch nicht der Fall. Chadwick und May haben in ihrer Untersuchung von Praktiken des eGovernment gezeigt, dass das möglicherweise existierende demokratische Potential des Internets faktisch marginalisiert wird. Statt dessen wird ein von Entscheidungsträgern bestimmtes Managementmodell der Interaktion durchgesetzt (Chadwick/May 2003). Auch fehlen in der EU Möglichkeiten zur tatsächlichen Beteiligung trotz gegenläufiger Rhetorik in der Regel (Monaghan 2007). Debatten bleiben meist auf Elitezirkel beschränkt (Kohler-Koch/Quittkat 2011). Partizipatorische Stufen im Sinne von Arnstein werden so nicht erreicht.

Mit Web-2.0-Medien entstehen also kaum partizipative Strukturen im Sinne eines Einflusses auf die Ergebnisse von Entscheidungsprozessen. Es kommt, im Gegensatz zur gelegentlich unterstellten demokratischen Medialität, 
nicht zu einer BürgerInnenbeteiligung und daher auch zu keiner Beteiligung an Macht. In Anlehnung an Arnstein ist eher ein Massentherapieprozess zu vermuten, der Ausdruck einer Kontrolle mittels Chiffren im Sinne von Deleuze (1990) ist.

Nun atmet Arnsteins Leiter den Geist der 1960er-Jahre. Kritisiert oder weiter entwickelt wird das Modell eher selten. Collins und Ison (2006) schlagen vor, den Partizipationsbegriff durch den des sozialen Lernens in politischen Kontexten zu ersetzen. Und Tritter und McCallum (2006) bevorzugen die Metapher des Mosaiks anstelle der von Arnstein vorgeschlagenen Leiter, um der Komplexität des Problems besser gerecht werden zu können. In beiden Fällen wird kein Bezug zu staatstheoretischen Überlegungen hergestellt.

Wenn aber Arnstein die Rechte von Bürgerinnen und Bürgern gegenüber einem Staat, der diese Rechte nicht respektiert, betont, wird damit das Ziel einer Minimalisierung des Staates und einer Maximierung des Einflusses der einzelnen Bürgerinnen und Bürger angestrebt. Es handelt sich damit um ein libertäres Modell. Unklar bleibt allerdings, ob es sich um eine kompetitivoder solidarisch-libertäres Modell handelt, da nur die Konkurrenz zwischen Bürgerinnen, Bürgern und Staat, nicht aber die Konkurrenz zwischen Bürgerinnen diskutiert wird. Insofern aber von Arnstein eben eine Konkurrenz zwischen Bürgerinnen, Bürgern und Staat angenommen wird, handelt es sich offenbar eher um ein kompetitiv-libertäres Modell.

Das Konzept von Arnstein ist nie als komplexer Entwurf gemeint gewesen. Das ist mit dem Partizipationsbegriff anders, wenn er - und das ist sicher das relevanteste Verständnis - als politische Partizipation konzipiert wird. Während bei Ansätzen, die sich im Kontext von Arnstein bewegen, das zugrunde liegende Staatsmodell meist nicht expliziert wird, ist das bei Wagner u.a. anders, die explizit (2011: 2) an die Theorie der deliberativen Demokratie von Habermas anknüpfen. Dabei werden treffend zwei Probleme angesprochen, die bei Habermas nicht berücksichtigt werden: Zum einen die pädagogische Aufgabe der Erziehung zur Partizipation und zum anderen die entwicklungspädagogische Aufgabe der Entwicklung altersgerechter Bil- 
dungsanlässe. Auch die kommerziellen Interessen, die hinter vielen Web2.0-Medien stehen, werden im Anschluss an Jenkins berücksichtigt und ein reflektierender Zugang empfohlen.

Nicht berücksichtigt werden bei Wagner u.a. aber zwei Aspekte: Zum einen gibt es keinen Staat, in dem eine deliberativ zu nennende Demokratie existiert. Damit ist das Ziel der Partizipation nicht die Teilhabe am Leben in der existierenden Gesellschaft, sondern die Vorbereitung auf eine zukünftige Gesellschaft. Es handelt sich also um einen utopischen Entwurf. Der Unterschied zum in die medienpädagogische Debatte von Habermas übernommenen Empanzipationsbegriff, der an dieser Stelle lange Zeit das medienpädagogische Denken bestimmt hat, ist, dass mit dem Partizipationsbegriff nicht die offene Aufgabe der Emanzipation, sondern ein bestimmtes Modell in den Mittelpunkt gerückt wird. Das Modell der deliberativen Demokratie ist allerdings keinesfalls unumstritten (Ottmann 2006), und zwar nicht nur zwischen demokratietheoretischen Ansätzen, sondern auch innerhalb verschiedener Konzepte deliberativer Demokratie (Thaa 2006).

Das Konzept der deliberativen Demokratie ist eine Alternative zu anderen demokratischen Entwürfen wie dem demokratischen Sozialismus von Lorenzen (1980), der über das von Jörrissen und Marotzki rezipierte Konzept des Orientierungswissens ebenfalls Relevanz für die Medienpädagogik gewonnen hat, und es ist eine Alternative zu nicht-demokratischen Konzepten, die zumindest faktisch derzeit in vielen Ländern praktiziert werden. Der Diskurs zu diesen Alternativen wird aber suspendiert, wenn der Partizipationsbegriff als Normierung der Medienpädagogik ohne weitere Debatte an das Konzept der deliberativen Demokratie angehängt wird. Das kann aus Sicht von Rezipientinnen und Rezipienten kaum als demokratisch bezeichnet werden.

Darüber hinaus übersieht der Anschluss an das Konzept der deliberativen Demokratie einen aus medienpädagogischer Sicht relevanten Punkt: Die Entwicklung der Medien, die eigentlich gerade zum Anlass der Auseinan- 
dersetzung mit dem Partizipationsgedanken werden. Capurro schreibt dazu: „Für Habermas ist, so scheint es, die UN selbst der Ort der rationalen Diskussion, während die elektronischen Medien, zumindest teilweise, eine Bedrohung, ja Irreführung des rationalen Denkens darstellen." (Capurro 1996). Damit wird aber der Buchdruck, und nicht die öffentliche Äußerung, zum Maßstab gemacht und übergangen, dass der Begriff des Wissens durch den Umstand, dass der Begriff der Arbeit im Bereich der Ökonomie gegenüber dem Begriff der Information an Relevanz verloren hat (Barberi 2001), eine andere Bedeutung erhalten hat, so dass wohl eine Demokratietheorie auch als Medientheorie gefasst werden muss. Die Relevanz medialer Infrastrukturen etwa für das Verhältnis von Repräsentation und Partizipation (Barberi 2005) ist dabei aber als durchaus problematisierungsbedürftig und nicht einfach nur als vermittlungsbedürftig einzuschätzen.

Es wird auch nicht deutlich, wo denn der Unterschied zwischen Citizen Control und parlamentarischer Arbeit liegt - abgesehen von dem Umstand, dass Arnstein nicht vorgesehen hat, dass die „Citizens“ über den Umstand hinaus, dass sie eben Citizens sind (wie es dazu kommen soll, wird ebenfalls nicht thematisiert) eine Legitimation benötigen. Offenbar handelt es sich hier nicht um ein durchdachtes repräsentativ-demokratisches Modell.

Es scheint gelegentlich unklar zu sein, wann eigentlich eine Handlung nicht als Partizipation zu verstehen ist. Die einzige eindeutige Ausnahme ist offenbar das Regierungshandeln. Das ist in einer Demokratie allerdings erstaunlich. Mitglied einer Partei zu werden, Ämter einzunehmen, einen Wahlkampf zu organisieren etc. - das alles wird als Partizipation verstanden. Wenn aber das ganze Unterfangen erfolgreich ist, die Mehrheit gewonnen wird und die partizipierende Person z.B. zur Bürgermeisterin gewählt wird, partizipiert sie plötzlich nicht mehr - als ob nicht ihre Absicht die ganze Zeit schon gewesen wäre, diese Machtposition zu erreichen, und als ob nicht schon Parteimitglieder, etwa über Parteiprogramme, Parteitage, Fraktionssitzungen etc. erheblichen Einfluss auf die amtsführende Person hätten. 
Hier hilft auch die Grenze zwischen institutionalisierter und nichtinstitutionalisierter Willensbildung, die Habermas (1992) vorgeschlagen hat, nicht weiter. Mit der Unterscheidung von institutionalisiertem und nicht institutionalisiertem politischen Handeln kann ja nicht unterstellt werden, dass ein Mensch nicht zwischen beiden Positionen wechselt oder das politische Handeln ganz einstellt, etwa um sich der Partizipation an vermischten Meldungen (Bourdieu 1998) oder gar Klatsch und Tratsch, die in Web-2.0-Medien wohl die dominanten Inhalte darstellen, zu widmen. In beiden Fällen reicht jedenfalls der Ausdruck des politischen Handelns aus.

\section{Diskussion}

Man sollte sich nicht täuschen: Durch die Offenlegung von Machtstrukturen, etwa durch eine kritische Fremdreflexion oder eine ironisierende Selbstreflexion, werden gesellschaftliche Machstrukturen weder angegriffen noch angeeignet. Mit Partizipation im Sinne der Bürgerbeteiligung wird das Prinzip der staatlichen Steuerung daher nicht suspendiert oder auch nur reduziert. Das mit dem Partizipationsbegriff zum Ausdruck gebrachte Ziel ist es dann nicht, staatliche Macht zugunsten individueller Macht im Sinn einer Selbstbefreiung des Menschen zu minimieren, sondern die staatliche Steuerung zu übernehmen. Damit werden bestehende Machtstrukturen anerkannt und fortgeschrieben. Dass dies schon bei Arnstein nicht anders gemeint war, wird daran sichtbar, dass sie die Stufen der Leiter auf die zu ihrer Zeit bestehenden politischen Institutionen in den USA bezogen hat.

Wenn das als sinnvoll gesehen wird, wäre es nötig, eine analoge Struktur für die jeweiligen lokalen politischen Institutionen zu entwickeln und ausgehend davon dem einzelnen Menschen die Kompetenz zur politischen Performanz zu vermitteln. Damit wäre für die Medienkompetenzvermittlung die handlungsorientierte Produktorientierung mehr mit der politischen Handlungsfähigkeit aufzuladen - was keine neue Idee ist, sondern mit der Gestaltung von Medien über das Mediensystem hinaus (Baacke 1997) im Medienkompetenzbegriff bereits mitgedacht ist. Damit könnte zumindest die Mög- 
lichkeit der Aneignung einer politischen Handlungfähigkeit versucht werden.

Nun wird mit der Verbreitung des Internet immer wieder die Annahme verbunden, dass Partizipation im Sinne einer Teilhabe an machtförmigen Entscheidungsprozessen ermöglicht wird. Auch die Studie des JFF argumentiert, dass eine Einübung in die Partizipation durch die Verwendung des Web 2.0 aus diesem Grund erstrebenswert sei. Ob dafür Web-2.0-Medien unbesehen geeignet sind, wird allerdings durchaus zu Recht bezweifelt (Gesert 2012). Angeregt durch den von Sesink (2004) entwickelten Simmulationsbegriff ließe sich hier durchaus Potential sehen, wenn nicht kommerzielle Produkte (Facebook, Google) herangezogen werden, sondern die Gestaltung von Software und die eigene Kontrolle der Server stärker in den Mittelpunkt rückt.

Eine medienpädagogisch sinnvolle Verwendung des Partizipationsbegriffs konnte damit nicht identifiziert werden. Aus medienpädagogischer Sicht ist allerdings klar geworden, dass mit dem Partizipationbegriff kaum die Emanzipation des Menschen aus bestehenden Machstrukturen oder ökonomischen Zwängen gemeint ist. Damit ist klar, dass mit dem Partizipationsbegriff nicht mehr eine mögliche Verbesserung unmenschlicher gesellschaftlicher Strukturen, sondern eine Disziplinierung und Kontrolle des Einzelnen zum Zwecke der Einpassung in solche Strukturen im Mittelpunkt steht.

Der Partizipationsbegriff ist so gesehen ein konservativer und nahezu antidemokratischer Begriff, mit dem die ökonomische und politische Einordnung des Menschen in bestehende gesellschaftliche Strukturen so in den Mittelpunkt rückt, dass die kompetitive Durchsetzung von Interessen in beiden Hinsichten entscheidend ist. Pateman (1970, S. 68 ff.) hat das als Pseudopartizipation bezeichnet.

So gesehen handelt es sich bei der Partizipation mit Web-2.0-Medien um ein Oxymoron: Krieg ist Frieden, Freiheit ist Sklaverei, Unwissenheit ist Stärke (George Orwell, 1984) - und Partizipation mit Web-2.0-Medien ist Demokratie und Freiheit. 
Partizipation erscheint dann nur noch als Anpassungsbildung, als Disziplinierungsmaßnahme, die den Mensch als brauchbares Werkzeug zum Ziel hat. Diese Struktur steht im Gegensatz zum Freiheitsgedanken. Die Vorstellung der Freiheit des Menschen kann allerdings, wie Reichenbach (1999) gezeigt hat, nicht mehr auf das sich souverän selbst bestimmende autonome Subjekt bauen: „Das spätmoderne Selbst muß seine Freiheit nun größtenteils praktizieren, obwohl ihm das Telos eines gemeinsamen Sinnhorizonts abhanden gekommen ist. Es konstituiert sich zum Subjekt nun vielmehr ohne eschatologische Hoffnung, sondern mit dem kontextgebundenen und (immer nur) situativen Motiv, seine Selbstachtung und Integrität gegen dissoziierende Kräfte zu verteidigen“ (Reichenbach 1999: 469). Anders gesagt, kann dem Selbst das Erlösungsversprechen nicht mehr mit dem Begriff des souveränen, autonomen und sich selbst transparenten Subjekts gegeben werden. Der Umstand, dass sich die Freiheit des Menschen nicht positiv bestimmen lässt und sie damit auch nicht als Kompetenz, Ziel oder Ideal ausgewiesen werden kann, führt Reichenbach zu der Bemerkung: „Erziehung handelt in diesem Sinne von der Zumutung an den „Zögling“, zunehmend so zu handeln, als ob er autonom wäre" (Reichenbach 1999: 366).

Erziehung handelt damit weniger von einer unbekannten Zukunft, und mehr von einer gegenwärtigen Praxis. Es geht also weniger um eine mögliche, aber empirisch dann doch eher selten beobachtbare Erlösung, sondern darum, jetzt zumindest so zu tun, als ob man erlöst wäre.

Mit dem Emanzipationsbegriff, der während der Entstehung der Medienpädagogik prägend war, rückt aber die bessere Gesellschaft nur in der Zukunft in den Blick, weil es keine wahre Emanzipation in einer falschen Gesellschaft gibt. Diese Perspektive erscheint aber zunehmend unplausibel, weil mit der durch die Computertechnologie induzierten Punktzeit (Gendolla 1987) eine Gegenwartsorientierung in den Mittelpunkt rückt, die eben keine zukünftig bessere Gesellschaft, sondern das gegenwärtige bessere Leben in den Mittelpunkt rückt. Dieser medieninduzierten Tendenz entspricht die Beschreibung von Reichenbach. Das Selbst, dass sich so verhält, als wäre es 
autonom, kann daher auch als Simulation des autonomen Subjekts angeschrieben werden.

Es geht also darum, jetzt an einer besseren Gesellschaft zu partizipieren. In diesem Sinne könnte der Partizipationsbegriff den Emanzipationsbegriff als nicht normierende Orientierung für die Medienkompetenzvermittlung ablösen. Denn im Gegensatz zu Wagner et. al., die Partizipation als normatives Konzept ausgewiesen haben, ist die Möglichkeit einer solchen Norm nicht anzunnehmen.

Partizipative Medienkompetenz würde dann heißen, dass die Menschen dazu aufgefordert werden, so zu tun, als ob sie mit Medien autonom handeln könnten - wohl wissend, dass sie das nie erreichen können. Dabei bleiben sie mit Reichenbach immer Diltettanten, haben aber durchaus auch die möglicherweise günstigere Option, sich als Narren zu gebärden und das Autonomiespiel zu spielen.

Hierzu ist derzeit erstens nicht die Marktteilnahme an einer konkurrenzlibertären Ökonomie zum Maßstab zu machen, sondern die Freiheit der Wahl zu bedenken (die allerdings in vielen Fällen ohne Zweifel erst einmal zu einer solchen werden müsste, wie Niesyto (etwa 2009) zu Recht immer wieder betont). Wird die Freiheit der Wahl nicht bedacht, kommt es wieder zu einer Normierung, die es nicht einmal mehr möglich macht so zu tun, als ob man frei handeln könne. Daher muss auch die Freiheit der Entscheidung für den Marktaustritt eingeschlossen werde, d.h. die Freiheit, sich für ein Leben am Existenzminimum zu entscheiden. Ein Gedanke, der nicht einmal neoliberalen Ideen widerspricht, war es doch gerade Hayek, der die staatliche Sicherstellung des Existenzminimums gefordert hat - allerdings in einer zu minimalistischen Variante, mit der die Konkurrenz eben nicht verlassen wird (Zintl 2000).

Zweitens gilt es, in kritischer Absicht mediale Alternativen erfahrbar zu machen und zu zeigen. Es genügt nicht, nur Web-2.0-Medien kennenzulernen. Hier wird wieder einmal deutlich, dass eine Restriktion medienpädagogischer Inhalte auf eine akzidentielle Querschnittsmaterie zu kurz greift. Eine 
sich als Wissensgesellschaft begreifende Mediendemokratie bedarf eben auch einer Medienkunde als schulischem Gegenstand.

Drittens gilt es, realistische (Medien-)Utopien als Alternativen zu veranschaulichen. Die Veranschaulichung von realistischen Medienutopien durch die Aufforderung zur Konstruktion entsprechender Simulationen in den Diskurs zu bringen und es damit zu ermöglichen, so zu tun als ob man daran partizipieren würde, ist eine durchaus veritable Herausforderung für die Medienpädagogik.

\section{Literatur}

Arnstein, S. R. (1969). A Ladder of Citizen Participation. JAIP (35) 4, 216-224.

Baacke, D. (1997). Medienpädagogik, Tübingen: Niemeyer.

Bourdieu, P. (1998). Über das Fernsehen. Suhrkamp, Frankfurt a. M. 1998.

Capurro, R. (1996). Informationsethik nach Kant und Habermas. http://www.capurro.de/graz.html, Zugegriffen: 29.3.2013.

Barberi, A. (2001): Die symbolischen Ordnungen des Politischen. Gezeit (2), 5-9.

Barberi, A. (2005): Mediale Politiken. Über das Programm der kommenden medienwissenschaftlichen Demokratietheorie. In D. Gethmann, D.; M. Stauff (Hrsg.), Politiken der Medien (S. 57-76). Zürich/Berlin: Diaphanes.

Chadwick, A. \& May, C. (2003): Interaction between States and Citizens in the Age of the Internet: "e-Government" in the United States, Briain, and the European Union . doi: 10.1111/1468-0491.00216 .

Collins, K. \& Ison, R. (2006). Dare we jump off Arnstein's ladder? Social learning as a new policy paradigm. Proceedings of Participatory Approaches in Science \& Technology Conference, 4-7 June 2006. Edinburgh.

Deleuze, G. (1990). Postskriptum über die Kontrollgesellschaften. http://www.nadir.org/nadir/archiv/netzkritik/postskriptum.html, Zugegrif- 
fen: 1.2.2013.

Dewey, J. (2000). Demokratie und Erziehung. Eine Einleitung in die pphilosphische Pädagogik. Herausgegeben von J. Oelkers. Weinheim: Beltz.

Dzierzbicka, A. (2006). Vereinbaren statt anordnen - Neoliberale Gouvernementalität macht Schule. Wien: Löcker Verlag.

EU High Level Group of Experts on Literacy (2012). RoF 1 NI 5 snaeporue, Act now eth DLROW SI DRAH. http://ec.europa.eu/education/literacy/what-eu/high-levelgroup/documents/literacy-final-report_en.pdf, Zugegriffen: 4.1.2013.

Europäisches Parlament (2013). Beschluss Nr. 1093/2012/EU des europäischen Parlaments und des Rates vom 21. November 2012 über das Europäische Jahr der Bürgerinnen und Bürger. uri=OJ:L:2012:325:0001:0008:DE:PDF.

European Commission (2010). Europe 2020. A strategy for smart, sustainable and inclusive growth. uri=COM:2010:2020:FIN:EN:PDF.

Foucault, M. (1992). Andere Räume. http://www.uniweimar.de/cms/uploads/media/Foucault_AndereRaeume_01.pdf, Zugegriffen: 6.7.2011.

Gendolla, P. (1987). Auf dem Weg in die Punktzeit. In: Bamm, A. (u.a. Hrsg.): Technologische Zivilisation. München, 121-131.

Geser, H. (2012). Kakophonie und Selbstorganisation in der digitialen [sic] Agora. MedienPädagogik 21, http://www.medienpaed.com/21/geser1205.pdf, Zugegriffen: 5.3.2013.

Gertenbach, L. (2007). Die Kultivierung des Marktes: Foucault und die Gouvernementalität des Neoliberalismus, Berlin: Parodos.

Grell, P.; Rau, F. (2011). Partizipationslücken - Social Software in der Hochschullehre. MedienPädagogik 21, http://www.medienpaed.com/21/grell_rau1111.pdf, Zugegriffen am: 12.3.2013. 
Gruppe hochrangiger Sachverständiger der EU für Schriftsprachkompetenz (2012). rüF nedej 5. reäporuE tsi eid tleW rewhcs uz nreffiztne. http://ec.europa.eu/education/literacy/what-eu/high-level-group/documents/executivesummary_de.pdf, Zugegriffen am 4.1.2013.

Gruschka, A. (2002). Didaktik. Das Kreuz mit der Vermittlung. Elf Einsprüche gegen den didaktischen Betrieb. Wetzlar: Büchse der Pandora.

Hayek, F. A. Von (1960). The constitution of liberty. Chicago: Chicago University Press.

Kirk, S.(2009): Mündliche Leistungen. Ein wichtiger Bestandteil der Leistungsbeurteilung. Grundschule 41, 44-46 .

Klafki, W. (1985): Neue Studien zur Bildungstheorie und Didaktik. Weinheim: Beltz.

Kohler-Koch, B. \& Quittkat, C. (2011): Die Entzauberung partizipativer Demokratie. Zur Rolle der Zivilgesellschaft bei der Demokratisierung von EU-Governance. Frankfurt am Main: Campus.

Lohmann, I. (2010). Bildung am Ende der Moderne. Beiträge zur Kritik der Privatisierung des Bildungsweses. Hamburg: Universität Hamburg. http://www.erzwiss.uni-hamburg.de/Personal/Lohmann/Privatisierungskritik/E-Book.pdf, Zugegriffen am 15.3.2013.

Lorenzen, P. (1980). Versuch einer wissenschaftlichen Grundlegung des Demokratischen Sozialismus. In Thomas Meyer (Hrsg.), Demokratischer Sozialismus - Geistige Grundlagen und Wege in die Zukunft (S. 29-41). München: Schweitzer.

Meder, N. (1998). Neue Technologien und Erziehung/Bildung. In M. Borelli \& J. Ruhloff, Deutsche Gegenwartspädagogik Band III (S. 26-40), Hohengehren: Schneider.

Mehr, C. (2010). Fallrekonstruktive Erforschung von Lehrer- und Schülerfragen im Unterrichtsgespräch. Zeitschrift für Geschichtsdidaktik 9 (Jahresband 2010), 191-205.

Milbrath, L. (1965). Political Participation. How and why do People get In- 
volved in Politics. Chicago: Rand McNally.

Mitgutsch, K. (2008). Lernen durch Enttäuschung. Skizze eines pädagogischen Umlernvollzugs. http://othes.univie.ac.at/3663/1/2008-1221_9908011.pdf, Zugegriffen am: 1.9.2009.

Monaghan, E. (2007). Civil Society, Democratic Legitimacy and the European Union: democratic linkage and the debate on the future of the EU . Nottingham: University of Nottingham.

Nickel, R. (2004). Beurteilung und Bewertung "mündlicher Mitarbeit". Der altsprachliche Unterricht Latein, Griechisch, 47, 2-7.

Niesyto, H. (2009): Medien, soziale Benachteiligung und soziale Distinktion. Medienpaed (17), http://www.medienpaed.com/17/niesyto0906.pdf, Zugegriffen am: 12.12.2012.

Pateman, C. (1970). Participation and democratic theory. Cambridge, Eng.: Cambridge University Press.

Panke, S. (2007): Unterwegs im Web 2.0: Charakteristiken und Potenziale. http://www.e-teaching.org/didaktik/theorie/informelleslernen/Web2.pdf, Zugegriffen am: 2.4.2013.

Wijnen, C. (2012): Community Medien: Orte der Partizipation, politischen Bildung und Medienkompetenzförderung. Medienimpulse (1), http://www.medienimpulse.at/articles/view/416, Zugegriffen am: 13.3.3013.

Ottmann, H. (2006): Liberale, republikanische, deliberative Demokratie. Synthesis Philosophica 42, 315-325.

Mückel, W. (2011). Wer fragt, führt das Gespräch. Deutschunterricht (64) 3, 4-11.

Sesink, W. (2004). In-formatio. Die Einbildung des Computers. Beiträge zur Theorie der Bildung in der Informationsgesellschaft. Berlin u.a.: Lit.

Swertz, C. (2000): Computer und Bildung. Eine medienanalytische Untersuchung der Computertechnologie in bildungstheoretischer Perspektive. Bielefeld: Universität Bielefeld. 
Swertz, C. (2006): Neue Medien. In Schirlbauer, A.; Dzierzbicka, A. (Hrsg.), Pädagogisches Glossar der Gegenwart (S. 71-79). Wien: Löcker.

Thaa, W.: Rationalität und Repräsentation in der deliberativen Demokratietheorie von Jürgen Habermas. Arbeitspapier des Teilprojekts C 7 im SFB 600. Trier: Universität Trier.

Tritter, J. Q. \& McCallum, A. (2006). The snakes and ladders of user involvement: moving beyond Arnstein. Health Policy (76), 156-168.

VHS Münster (2012): Bürgerfunk-Zertifikat für Personen mit Vorkenntnissen - Bürgerfunk-Retestierung. http://vhs.muenster.de/programm/buergerfunk-zertifikat-fuer-personen-mit-vorkenntnissen-buergerfunk-retestierung680030, Zugegriffen am 2.4.2013.

Wagner, U., Gehrlicher, P. \& Brüggen, N. (2011): Partizipation im und mit dem Social Web - Herausforderungen für die politische Bildung . Expertise für die Bundeszentrale für politische Bildung. München: JFF, http://www.jff.de/dateien/Expertise_Partizipation_Im_Social_Web.pdf, Zugegriffen am: 29.3.2013.

Zintl, R. (2000): Die liberträre Sozialstaatskritik bei von Hayek, Buchanan und Nozik. In Kersting, W. (Hrsg.), Politische Philosophie des Sozialsaates (S. 95-119), Weilerswist: Velbrück.

This work is licenced under the Creative Commons Attribution-Share Alike 3.0 Austria License. To view a copy of this licence, visit http://creativecommons.org/licenses/bysa/3.0/at/ or send a letter to Creative Commons, 171 Second Street, Suite 300, San Francisco, California 94105, USA. 